\title{
Karel Karták
}

On Carathéodory operators

Czechoslovak Mathematical Journal, Vol. 17 (1967), No. 4, 515-519

Persistent URL: http://dml.cz/dmlcz/100798

\section{Terms of use:}

(C) Institute of Mathematics AS CR, 1967

Institute of Mathematics of the Czech Academy of Sciences provides access to digitized documents strictly for personal use. Each copy of any part of this document must contain these Terms of use.

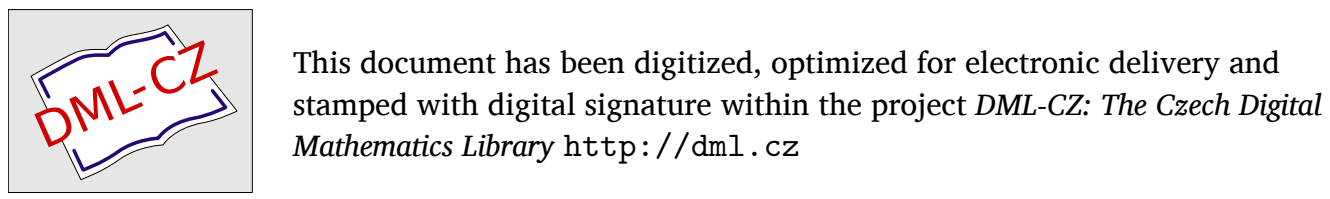




\title{
ON CARATHÉODORY OPERATORS
}

\author{
Karel Karták, Praha \\ (Received February 18, 1966)
}

1. This note represents a direct continuation of section 2 of [1]; definitions and notation of that paper are used here throughout. Our aim is to prove the following assertion:

Theorem A. Let $\mathrm{T}$ be a Carathéodory operator on $\mathrm{C}(I ; G)$. Then there exist classical Carathéodory operators $\mathrm{T}_{i}, i \in \mathscr{N}$ such that for each $\varphi \in \mathcal{C}(I ; G)$, $\lim \varrho\left(T_{i} \varphi, T \varphi\right)=0$.

2. First we prove some auxiliary results on polynomials in $n$ variables. Let $\pi_{n}(x)=$ $=x_{1} x_{2} \ldots x_{n}$. A real polynomial $P_{n}$ in $x_{1}, \ldots, x_{n}$ is said to be distinguished iff it is of the form

$$
P_{n}\left(x_{1}, \ldots, x_{n}\right)=\alpha \pi_{n}(x)+\sum_{j=1}^{n} \beta_{j} \frac{\pi_{n}(x)}{x_{j}}+\sum_{\substack{j, k=1 \\ j<k}}^{n} \gamma_{j k} \frac{\pi_{n}(x)}{x_{j} x_{k}}+\ldots+\sum_{j=1}^{n} \delta_{j} x_{j}+\varepsilon
$$

The same definition applies also to other sets of variables.

In what follows we prove that distinguished polynomials in $x_{1}, \ldots, x_{n}$ have with respect to $n$-dimensional cubes properties analogical to those of linear functions on segments.

We shall use the following convention: the symbol $\tilde{x}_{j}$ signifies that $x_{j}$ does not enter as a variable in our considerations.

3. Lemma. Let $P_{n}\left(x_{1}, \ldots, x_{n}\right)$ be a distinguished polynomial in $x_{1}, \ldots, x_{n}$, and let $\vartheta_{j_{1}}, \ldots, \vartheta_{j_{r}}, 1 \leqq r \leqq n$, be real numbers. Then $P_{n}\left(x_{1}, \ldots, \vartheta_{j_{1}}, \ldots, \vartheta_{j_{r}}, \ldots, x_{n}\right)$ is a distinguished polynomial in $x_{1}, \ldots, \tilde{x}_{j_{1}}, \ldots, \tilde{x}_{J r}, \ldots, x_{n}$.

Proof. It is sufficient to prove this for $r=1$, but then is it obvious.

4. Put $K=\langle 0,1\rangle \times \ldots \times\langle 0,1\rangle$ ( $n$ times), $N=2^{n}$, and given $j \in\{1, \ldots, n\}$, $\vartheta \in \mathscr{R}$, let $\left\{x_{j}=\vartheta\right\}=\left\{\left[x_{1}, \ldots, x_{n}\right] \in \mathscr{R}^{n} ; x_{j}=\vartheta\right\}$. Let $\left\{j_{1}, \ldots, j_{r}\right\}$ be a non-empty 
subset of $\{1, \ldots, n\}$, and let $\vartheta_{k}=0$ or 1 for $k=1, \ldots, r$; then the $(n-r)$-dimensional sides of $K$, denoted $K\left\{x_{j_{1}}=\vartheta_{1}, \ldots, x_{j_{r}}=\vartheta_{r}\right\}$, are defined by the formula $K \cap$ $\cap\left\{x_{j_{1}}=\vartheta_{1}\right\} \cap \ldots \cap\left\{x_{j_{r}}=\vartheta_{r}\right\}$. If $r=n$, we get the vertices of the cube $K, N$ in number; otherwise we have positive-dimensional sides of $K$, considered in the sequel as lower- dimensional cubes.

5. Lemma. Let $v_{j}, j=1, \ldots, N$, denote the vertices of $K$, and let $a_{j} \in \mathscr{R}$ for $j=$ $=1, \ldots, N$. There exists one and only one distinguished polynomial $P_{n}$ in $x_{1}, \ldots, x_{n}$ such that

$$
P_{n}\left(v_{j}\right)=a_{j}, \quad j=1, \ldots, N
$$

Proof. If we put $\varepsilon=P_{n}(0,0, \ldots, 0), \delta_{1}=P_{n}(1,0, \ldots, 0)-\varepsilon$, etc., then it is from (2.1) clear that all coefficients may be successively determined for (5.1) to be satisfied.

The numbers $a_{j}$ corresponding to the vertices $v_{j}$ of $K, j=1, \ldots, N$, are considered to be fixed in some further sections; thus, the above polynomial will be denoted simply by $P_{n}\left(x_{1}, \ldots, x_{n} ; K\right)$.

6. Lemma. Let $1 \leqq r \leqq n-1$, and let $Q=K\left\{x_{j_{1}}=\vartheta_{1}, \ldots, x_{j_{n-r}}=\vartheta_{n-r}\right\}$ be an $r$-dimensional side of $K$. Then

$$
P_{n}\left(x_{1}, \ldots, x_{n} ; K\right) \mid Q=P_{r}\left(x_{1}, \ldots, \tilde{x}_{j_{1}}, \ldots, \tilde{x}_{j_{n-r}}, \ldots, x_{n} ; Q\right)
$$

Proof. In virtue of Lemma 3, the left-hand side is a distinguished polynomial in $x_{1}, \ldots, \tilde{x}_{j_{1}}, \ldots, \tilde{x}_{j_{n-r}}, \ldots, x_{n}$ taking on the prescribed values. Now we apply Lemma 5 .

7. Lemma. For each $j=1, \ldots, n$, we have

$$
\begin{gathered}
P_{n}\left(x_{1}, \ldots, x_{n} ; K\right)=\left(1-x_{j}\right) P_{n-1}\left(x_{1}, \ldots, \tilde{x}_{j}, \ldots, x_{n} ; K\left\{x_{j}=0\right\}\right)+ \\
+x_{j} P_{n-1}\left(x_{1}, \ldots, \tilde{x}_{j}, \ldots, x_{n} ; K\left\{x_{j}=1\right\}\right)
\end{gathered}
$$

Proof. In virtue of Lemma 5 it is sufficient to note that the right-hand side is a distinguished polynomial in $x_{1}, \ldots, x_{n}$ taking on the prescribed values.

8. Lemma. For each $\xi=\left[\xi_{1}, \ldots, \xi_{n}\right] \in K$, we have

$$
\min \left\{a_{1}, \ldots, a_{N}\right\} \leqq P_{n}\left(\xi_{1}, \ldots, \xi_{n} ; K\right) \leqq \max \left\{a_{1}, \ldots, a_{N}\right\}
$$

Proof. For $n=1$ is it clear. Suppose the assertion is true for $n-1$. As a consequence of (7.1), we have

$$
\begin{gathered}
P_{n}\left(\xi_{1}, \ldots, \xi_{n} ; K\right)=\left(1-\xi_{n}\right) P_{n-1}\left(\xi_{1}, \ldots, \xi_{n-1} ; K\left\{x_{n}=0\right\}\right)+ \\
+\xi_{n} P_{n-1}\left(\xi_{1}, \ldots, \xi_{n-1} ; K\left\{x_{n}=1\right\}\right)
\end{gathered}
$$


Using induction assumption, we get from it e.g.

$$
\begin{gathered}
P_{n}\left(\xi_{1}, \ldots, \xi_{n} ; K\right) \leqq\left(1-\xi_{n}\right) \max \left\{a_{1}, \ldots, a_{N}\right\}+\xi_{n} \max \left\{a_{1}, \ldots, a_{N}\right\}= \\
=\max \left\{a_{1}, \ldots, a_{N}\right\}
\end{gathered}
$$

9. Let $i \in \mathscr{N}$. The division $\mathscr{D}_{i}^{1}$ of $\mathscr{R}$ of the $i$-th rank is the set of all points of the form $k 2^{-i}$, where $i \in \mathscr{N}$ and $k$ is an integer. The division $\mathscr{D}_{i}^{n}$ of $\mathscr{R}^{n}$ of the $i$-th rank is the set $\mathscr{D}_{i}^{1} \times \ldots \times \mathscr{D}_{i}^{1}\left(n\right.$ times); from now on, we write merely $\mathscr{D}_{i}$, instead of $\mathscr{D}_{i}^{n}$. It is clear that $\mathscr{D}_{i}$ induces a decomposition of $\mathscr{R}^{n}$ into non-overlapping cubes, with the edge $2^{-i}$ each. Let $\left\{\mathscr{D}_{i}\right\}$ denote the set of all these cubes.

10. After the preliminaries, we are going to prove the theorem stated in section 1 Here, $P\left(x_{1}, \ldots, x_{n} ; a_{1}, \ldots, a_{N}\right)$ is a new notation for the polynomial $P_{n}$ of Lemma 5 Also, if $f$ denotes a point of $\mathscr{R}^{n}$ or a vector function, then $f^{(j)}, j=1, \ldots, n$, denotes the $j$-th component of it. The vector-space operations on $\mathscr{R}^{n}$ are denoted in the usual manner.

Let $i \in \mathscr{N}$. For each $v \in \mathscr{D}_{i} \cap G$, let $f_{v}$ be a vector function on $I$ such that $\left[f_{v}\right]=$ $=\mathrm{T} \hat{v}$. Let us now define a vector function $f_{i}$ on $I \times G$, generating a classical Carathéodory operator. The construction will be carried out for $f_{i}^{(1)}$ only; for other components of $f_{i}$ is it analogous.

Let $K_{0} \in\left\{\mathscr{D}_{i}\right\}$ be such that $K_{0}=\left\{x \in \mathscr{R}^{n} ; 0 \leqq x-v \leqq 2^{-i}\right\}$, for some $v \in \mathscr{D}_{i}$. Suppose that

$$
K_{0} \cap G \neq \emptyset
$$

and let $v_{1}=v, v_{2}, \ldots, v_{N}$ denote the vertices of $K$. Let us define the finite functions $a_{j} \mid I, j=1, \ldots, N$, as follows: if $v_{j} \in G$, put $a_{j}(t)=f_{i, v_{j}}^{(1)}(t)$; otherwise put $a_{j}(t)=v_{j}^{(1)}$. Now, let for $t \in I, x \in K_{0}$

$$
f_{i}^{(1)}(t, x)=P\left(2^{i}\left(x^{(1)}-v_{1}^{(1)}\right), \ldots, 2^{i}\left(x^{(n)}-v_{1}^{(n)}\right) ; a_{1}(t), \ldots, a_{N}(t)\right)
$$

and similarly for $f_{i}^{(j)}, j=2, \ldots, n$, and all cubes of $\left\{\mathscr{D}_{i}\right\}$, satisfying (10.1). It follows from Lemma 6 that using $(10.2), f_{i}$ may be well-defined on $I \times G$; we show that it generates a Carathéodory operator, i.e., it satisfies the conditions of Theorem $(2,4)$ in [1].

Let $x \in G$. Then $f_{i}(., x)$ is measurable on $I$, as a "polynomial" of measurable functions. Let $t \in I$. Then $f_{i}(t,$.$) is continuous on G$, as a simple consequence of (10.2) and Lemma 6.

We are going to prove that, for each $\varphi \in \mathcal{C}(I ; G), \lim \varrho\left(\left[f_{i} \circ \varphi\right], \mathrm{T} \varphi\right)=0$; thus, to prove the theorem, it suffices to put $\mathrm{T}_{i} \varphi=\left[f_{i} \circ \varphi\right]$.

Let $\varphi \in C(I ; G)$. Given $i \in \mathscr{N}$, there exists $\delta_{i}>0$ such that

$$
\left|t-t^{\prime}\right|<\delta_{i}, \quad t, t^{\prime} \in I \Rightarrow\left|\varphi(t)-\varphi\left(t^{\prime}\right)\right|<2^{-i}
$$


Let $\tau_{0}=\tau<\tau_{1}<\ldots<\tau_{k}=\tau+\alpha, \max \left\{\tau_{j}-\tau_{j-1} ; j=1, \ldots, k\right\}<\delta_{i}$. In virtue of $(10.3)$, for each $j=1, \ldots, k$, there exists $s_{i}(j) \in \mathscr{D}_{i}$ such that

$$
t \in\left\langle\tau_{j-1}, \tau_{j}\right\rangle \Rightarrow\left|\varphi(t)-s_{i}(j)\right|<2^{-i}
$$

Let us define $\widehat{s}_{i}\left|I=\hat{s}_{i}(1)\right|\left\langle\tau_{0}, \tau_{1}\right) \oplus \ldots \oplus \hat{s}_{i}(k) \mid\left\langle\tau_{k-1}, \tau_{k}\right\rangle$. From (10.4) we infer that $s_{i}$ converge to $\varphi$ uniformly on $I$. For each $i \in \mathscr{N}$, it holds

$$
\varrho\left(\mathrm{T} \varphi, \mathrm{T}_{i} \varphi\right) \leqq \varrho\left(\mathrm{T} \varphi, \mathrm{T}_{s_{i}}\right)+\varrho\left(\mathrm{T}_{s_{i}}, \mathrm{~T}_{i} s_{i}\right)+\varrho\left(\mathrm{T}_{i} s_{i}, \mathrm{~T}_{i} \varphi\right)
$$

As a consequence of the definition of $\mathrm{T}_{i}, \varrho\left(\mathrm{T}_{i}, \mathrm{~T}_{i} s_{i}\right)=0$. In virtue of Lemma $(2,6)$ of $[1], \lim \varrho\left(T \varphi, T_{i}\right)=0$. Thus, it is sufficient to prove that

$$
\lim \varrho\left(\mathrm{T}_{i} s_{i}, \mathrm{~T}_{i} \varphi\right)=0
$$

Let $v=3^{n}$, and let $\varepsilon_{1}, \ldots, \varepsilon_{v}$ be the points of $\mathscr{R}^{n}$ such that $\varepsilon_{k}^{(j)}, k=1, \ldots, v, j=$ $=1, \ldots, n$, equals to $-1,0$ or 1 , independently.

First we prove an estimation, which could easily be made more precise but which suffices for our purpose. In virtue of Lemma 8, we have for $i \in \mathscr{N}, t \in I$ and each $j=1, \ldots, n$

$(10.6)\left|f_{i}^{(j)}(t, \varphi(t))-f_{i}^{(j)}\left(t, s_{i}(t)\right)\right| \leqq \sum_{k, l=1}^{v}\left|f_{i}^{(j)}\left(t, s_{i}(t)+2^{-i} \varepsilon_{k}\right)-f_{i}^{(j)}\left(t, s_{i}(t)+2^{-i} \varepsilon_{l}\right)\right|$

Now, put $s_{i}(t)+2^{-i} \varepsilon_{k}=s_{i}(t ; k)$. The step functions $s_{i}(. ; k) \mid I$ thus defined evidently converge to $\varphi$ uniformly on $I$, for each $k=1, \ldots, v$.

We have from (10.6)

$$
\begin{aligned}
& \left|f_{i}(t, \varphi(t))-f_{i}\left(t, s_{i}(t)\right)\right|=\max \left\{\mid f_{i}^{(1)}(\ldots)-f_{i}^{(1)}(\ldots), \ldots\right\} \leqq \\
& \quad \leqq \max \left\{\sum_{k, l=1}^{v}\left|f_{i}^{(1)}\left(t, s_{i}(t ; k)\right)-f_{i}^{(1)}\left(t, s_{i}(t ; l)\right)\right|, \ldots\right\} \leqq \\
& \quad \leqq \sum_{\substack{k, l=1 \\
k<l}} \max \{\ldots\}=\sum_{\substack{k, l=1 \\
k<l}}^{v}\left|f_{i}\left(t, s_{i}(t ; k)\right)-f_{i}\left(t, s_{i}(t ; l)\right)\right|
\end{aligned}
$$

Hence we get

$$
\min \left(1,\left|\mathrm{~T}_{i} \varphi-\mathrm{T}_{i} s_{i}\right|\right) \leqq \sum_{\substack{k, l=1 \\ k<l}}^{v} \min \left(1,\left|\mathrm{~T}_{i} s_{i}(. ; k)-\mathrm{T}_{i} s_{i}(. ; l)\right|\right)
$$

so that

$$
\begin{gathered}
\varrho\left(\mathrm{T}_{i} \varphi, \mathrm{T}_{i} s_{i}\right) \leqq \sum_{\substack{k, l=1 \\
k<l}}^{v} \varrho\left(\mathrm{T}_{i} s_{i}(. ; k), \mathrm{T}_{i} s_{i}(. ; l)\right)= \\
=\sum_{\substack{k, l=1 \\
k<l}}^{v} \varrho\left(\mathrm{T} s_{i}(. ; k), \mathrm{T}_{i}(. ; l)\right) \leqq \sum_{\substack{k, l=1 \\
k<l}}^{v} \varrho\left(\mathrm{T} s_{i}(. ; k), \mathrm{T} \varphi\right)+ \\
+\sum_{\substack{k, l=1 \\
k<1}}^{v} \varrho\left(\mathrm{T} \varphi, \mathrm{T}_{s}(. ; l)\right)
\end{gathered}
$$

Now, $(10.5)$ follows in virtue of Lemma $(2,6)$ of $[1]$. This proves Theorem A. 
11. Theorem B. Let $\mathrm{T}$ be a Carathéodory operator on $\mathrm{C}(I ; G)$ and let $\left\{f_{i}\right\}$ be the sequence of vector functions on $I \times G$ defined in the proof of Theorem A. Suppose that there exists $N \subset I$ such that

$$
p(N)=0
$$

(11.2) $t \in I-N \Rightarrow f_{i}(t,$.$) converge uniformly on compact subsets of G$

Then $f \mid I \times G$, defined for $t \in I-N, x \in G$ as $f(t, x)=\lim f_{i}(t, x)$, satisfies the conditions (2.4.1), (2.4.2) of Theorem $(2,4)$ of [1], generating thus a classical Carathéodory operator. Further, $\mathrm{T}_{\varphi}=[f \circ \varphi]$ for each $\varphi \in C(I ; G)$.

Proof. From (11.2) we see that $f(t,$.$) is continuous on G$ for each $t \in I-N$. Further, $f(., x)$ is measurable on $I$ for each $x \in G$, as a limit a.e. of measurable functions. To prove the second assertion of this theorem, it is sufficient to note that $T \hat{\xi}=$ $=[f \circ \hat{\xi}]$ for each $\xi \in \bigcup_{i=1}^{\infty} D_{i} \cap G$; see Corollary $(2,7)$ in $[1]$.

12. The author believes that the assumptions of Theorem $B$ are fulfilled for each Carathéodory operator; to solve Problem $\mathrm{C}$ of [1], it would be enough to prove this hypothesis. In section 14, we prove that this is true for classical Carathéodory operators.

13. Lemma. Let $f$ be a finite continuous function on $\langle 0,1\rangle$. For each $i \in \mathscr{N}$, let $t_{0}, t_{1} \ldots, t_{2}$, where $0=t_{0}<t_{1}<\ldots<t_{2 i}=1$, denote the points of $\mathscr{D}_{i}^{1} \cap\langle 0,1\rangle$. Let $l_{i}\left(t_{k}\right)=f\left(t_{k}\right), k=0,1, \ldots, 2^{i}$, and let $l_{i}$ be linear on each $\left\langle t_{k-1}, t_{k}\right\rangle$. Then $l_{i}$ converge to $f$ uniformly on $I$.

Proof. Given $\varepsilon>0$, there exists $\delta>0$ such that $t_{1}^{\prime}, t_{2}^{\prime} \in\langle 0,1\rangle,\left|t_{1}^{\prime}-t_{2}^{\prime}\right| \leqq \delta \Rightarrow$ $\Rightarrow\left|f\left(t_{1}^{\prime}\right)-f\left(t_{2}^{\prime}\right)\right| \leqq \varepsilon$. For each $i \in \mathscr{N}$, let $t(i) \in \mathscr{D}_{i}^{1} \cap\langle 0,1\rangle$ be such that $|t-t(i)| \leqq$ $\leqq 2^{-i}$. Let $i_{0} \in \mathscr{N}$ be such that $2^{-i_{0}} \leqq \delta$. Now using linearity of $l_{i}$, we get for each $i \geqq i_{0}$ and $t \in I$ that $\left|f(t)-l_{i}(t)\right| \leqq|f(t)-f(t(i))|+f(t(i))|+| f(t(i))-l_{i}(t(i)) \mid+$ $+\left|l_{i}(t(i))-l_{i}(t)\right| \leqq \varepsilon+0+\varepsilon=2 \varepsilon$ which proves the lemma.

14. Theorem C. Let $\mathrm{T}$ be a classical Carathéodory operator on $\mathrm{C}(I ; G)$, represented by a vector function $f \mid I \times G$. Then, the assumptions of Theorem $B$ are fulfilled.

Proof. This is an easy consequence of the preceding lemma.

15. Corollary. Let $f$ be the vector function of Theorem $C$. Let $\lambda^{n}$ denote the Lebesgue measure on $R^{n}$. Then $f$ is measurable on $\left(I \times G, p \times \lambda^{n}\right)$.

\section{Reference}

[1] K. Karták: A generalization of the Carathéodory theory of differential equations, Czech. Math. Journal, 17 (92) (1967), 482-514.

Author's address: Praha 6 - Dejvice, Technická 5, ČSSR (Vysoká škola chemicko-technologická). 